\title{
Direct Superior Ophthalmic Vein Approach to Treat Anterior Condylar Confluence Dural Arteriovenous Fistula
}

\author{
Rasmiranjan Padhi, MD, PDCC ${ }^{1}$, Sathish Kandasamy, MD, DMํ․ Balasenthil Kumaran, DNB 2 , \\ Naci Kocer, $\mathrm{MD}^{3}$, Harshith Karmadhari, MD, DM ${ }^{4}$ \\ ${ }^{1}$ Department of Interventional Neuroradiology, G. Kuppuswamy Naidu Memorial Hospital, Coimbatore, India \\ ${ }^{2}$ Department of Neurosurgery, G. Kuppuswamy Naidu Memorial Hospital, Coimbatore, India \\ ${ }^{3}$ Division of Neuroradiology, Department of Radiology, Cerrahapasa Medical Faculty, Istanbul University, Istanbul, Turkey \\ ${ }^{4}$ Department of Radiology, St John's Medical College, Bengaluru, India
}

Dural arteriovenous fistulas (DAVFs) are acquired pathological arteriovenous connections involving vessels that usually supply the meninges. A DAVF in the region of the hypoglossal canal is a rare form of fistula that involves the anterior condylar confluence or anterior condylar vein. We report a case of hypoglossal canal DAVF that was successfully embolized transvenously through a superior ophthalmic vein (SOV) approach. After failed attempts through jugular access, our patient was treated by a unique percutaneous direct puncture approach through the SOV, achieving complete obliteration of the fistula. A step-by-step description of the endovascular technique was described. The clinical course was uneventful without any new neurologic deficit. The eye symptoms and third nerve palsy had completely resolved at the 3-month follow-up visit. Hypoglossal canal DAVFs are rare and may exhibit complex venous drainage patterns. Knowledge of the complex venous anatomy is essential for planning an alternative transvenous route if the standard approach is not feasible. Xper CT (Philips Healthcare, Best, The Netherlands) is an excellent tool for identifying the exact site of the fistula as well as for confirming a safe position of the catheter tip for successful occlusion of this complex dural AV fistula.

Key Words: Dural arteriovenous fistula; Hypoglossal; Embolization

\section{INTRODUCTION}

Dural arteriovenous fistulas (DAVFs) in the region of the hypoglossal canal $(\mathrm{HC})$ are a rare form of fistula that involves the anterior condylar confluence (ACC) or anterior condylar vein (ACV). The fistula location near the condylar confluence predisposes hypoglossal canal dural arteriovenous fistulas (HCDAVFs) to a complex venous network involving the orbital veins, cavernous sinus, dural sinuses, and the vertebral venous system. ${ }^{2}$ The standard approach for management of HCDAVFs is transvenous and/or transarterial embolization. When antegrade venous drainage of the fistula to the internal jugular vein (IJV) is restricted, then the drainage may be retrograde to the cavernous sinus and ophthalmic veins via the inferior petrosal sinus (IPS). In such scenarios, accessing the ACC through IJV is not possible, leaving the cavernous sinus as an alternative

\section{Correspondence to: Sathish Kandasamy, MD, DM Department of Interventional Neuroradiology, G. Kuppuswamy Naidu Memorial Hospital, Coimbatore 641037, Tamil Nadu, India \\ Tel: +91-9894384734 \\ Fax: +91-4224305352 \\ E-mail: sathisam@gmail.com}

Received: July 22, 2021

Revised: September 13, 2021

Accepted: September 29, 2021
Copyright $\odot 2021$ Korean Society of Interventional Neuroradiology This is an Open Access article distributed under the terms of the Creative Commons Attribution Non-Commercial License (http://creativecommons.org/licenses/by-nc/4.0) which permits unrestricted non-commercial use, distribution, and reproduction in any medium, provided the original work is properly cited.

pISSN 2093-9043 eISSN 2233-6273 
approach for embolization. Direct transorbital puncture of the superior ophthalmic vein (SOV) has been rarely reported and is mostly limited to the management of cavernous sinus DAVF. We present our experience in treating a case of ACC DAVF by direct transorbital puncture of the superior ophthalmic vein as an alternative endovascular approach.

\section{CASE REPORT}

A 55-year-old male was presented to our hospital with a 1-month history of left eye chemosis, proptosis, and third nerve paresis. His ophthalmological examination showed elevated intraocular pressure. Magnetic resonance imaging revealed a dilated right SOV with an engorged cavernous sinus. Magnetic resonance angiography showed an abnormal flow-related enhancement of the right cavernous sinus and IPS. A cerebral angiogram was carried out in the neurointerventional suite (Azurion Biplane; Philips Healthcare,
Best, The Netherlands). 3D rotational angiography and Xper CT imaging were also performed (20s DR-Head protocol, Xper CT; Philips Healthcare). The angiogram revealed right HCDAVF fed by the hypoglossal branches of the ascending pharyngeal artery and stylomastoid branch of the occipital artery. Retrograde venous drainage occurred predominantly through the IPS towards the cavernous sinus with cortical venous drainage into the superficial middle cerebral vein. The SOV was dilated, draining into the facial vein (Figs. 1A, $1 B, 2)$. No connection was noted between the ipsilateral IJV and the ACC. With the patient under general anesthesia, arterial access was achieved via the right common femoral artery. Venous access through the right femoral vein had been performed to attempt a transvenous approach. A 5F diagnostic catheter was placed into the right external carotid artery to perform control angiogram and road mapping imaging. The transarterial approach was not attempted as it carries a substantial risk of cranial nerve palsies with liquid embolic agents. A contralateral IPS approach to reach the
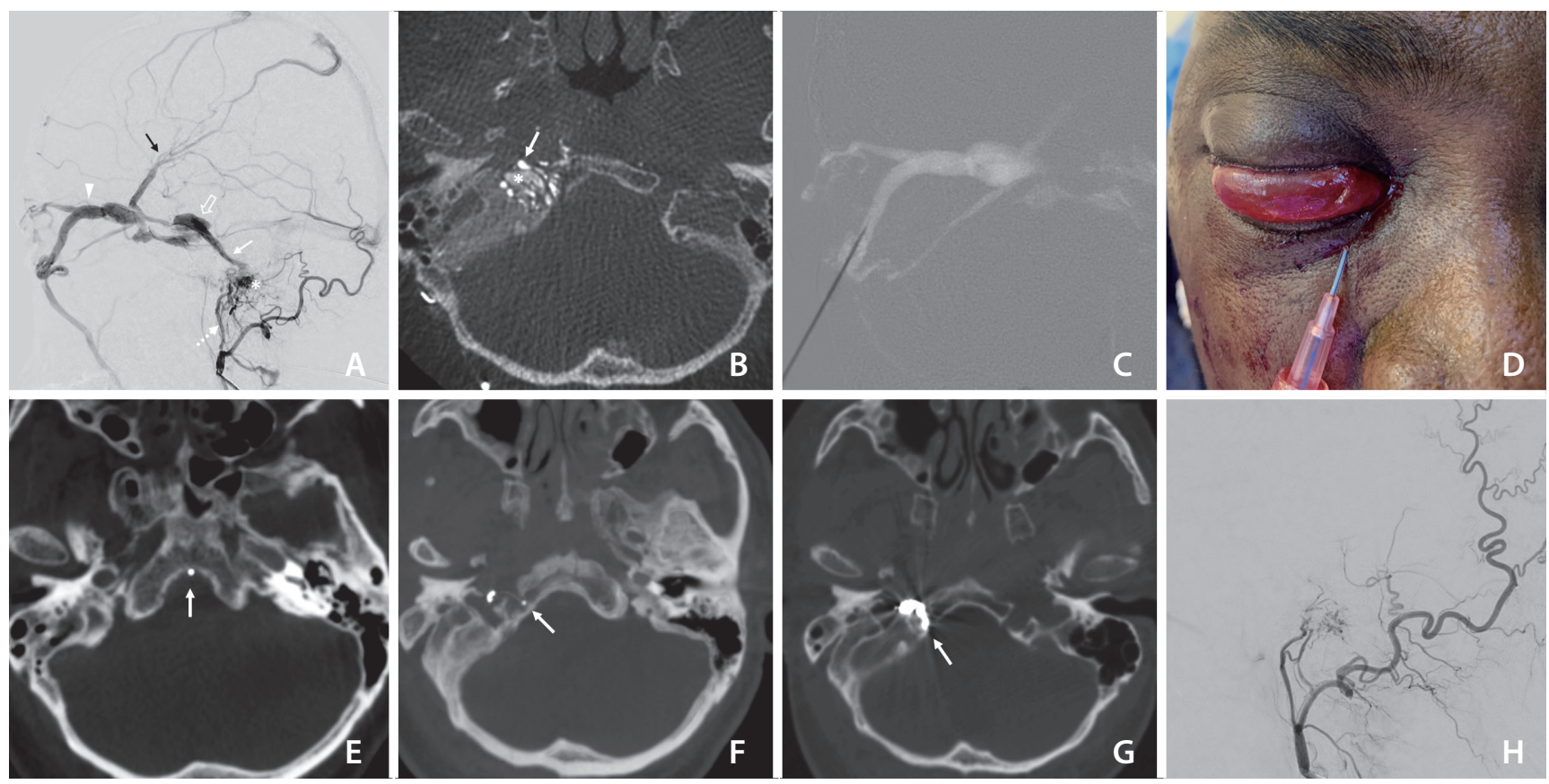

Fig. 1. Right ascending pharyngeal artery digital subtraction angiogram lateral projection (A) demonstrating hypoglossal canal dural arteriovenous fistula (asterisk) with arterial supply from neuromeningeal trunk of ascending pharyngeal artery (dashed arrow). Retrograde venous drainage is seen directed into the inferior petrosal sinus (arrow), cavernous sinus (open arrow), superior ophthalmic vein (SOV) (arrowhead) with cortical venous reflux (black arrow). (B) Axial 3D rotational angiography images show multiple arterial feeders (white arrow) and the dilated anterior condylar vein and venous pouch (asterisk) in the hypoglossal canal. (C, D) Roadmap guided puncture of anterior apsidal tribute of the SOV (inferomedial approach). (E) During the procedure, the catheter tip was placed inadvertently in the basilar plexus (arrow), which was checked with Xper CT (Philips Healthcare, Best, The Netherlands. (F) A safe position of the microcatheter tip (arrow) inside the fistulous pouch was confirmed with Xper CT. (G) Axial reconstruction of the post-coiling Xper CT scan shows coil mass inside the hypoglossal canal (arrow). Post embolization angiogram, lateral view (H) shows complete obliteration of the fistula. 
right cavernous sinus via inter-cavernous sinus failed. Venous access through the facial vein was also not possible because it drained to multiple small tortuous veins. Hence, it was decided to perform a direct percutaneous approach. The right eye region was prepared in a sterile fashion. The inferomedial approach was chosen and the medial third of the lower eyelid was the entry point. The eyeball was gently dislocated superiorly with the thumb. With the patient's head in a neutral position, a 20-gauge 2 inch puncture needle was advanced towards the extraconal portion of the SOV under roadmap guidance (Fig. 1C, D). When the puncture needle reached the SOV in the lateral roadmap, the inner stylet was taken out and backflow of blood was observed. The outer cannula of the puncture needle was fixed as access and attached to a rotating hemostatic valve. After stabilizing the cannula, venography was performed to confirm the position

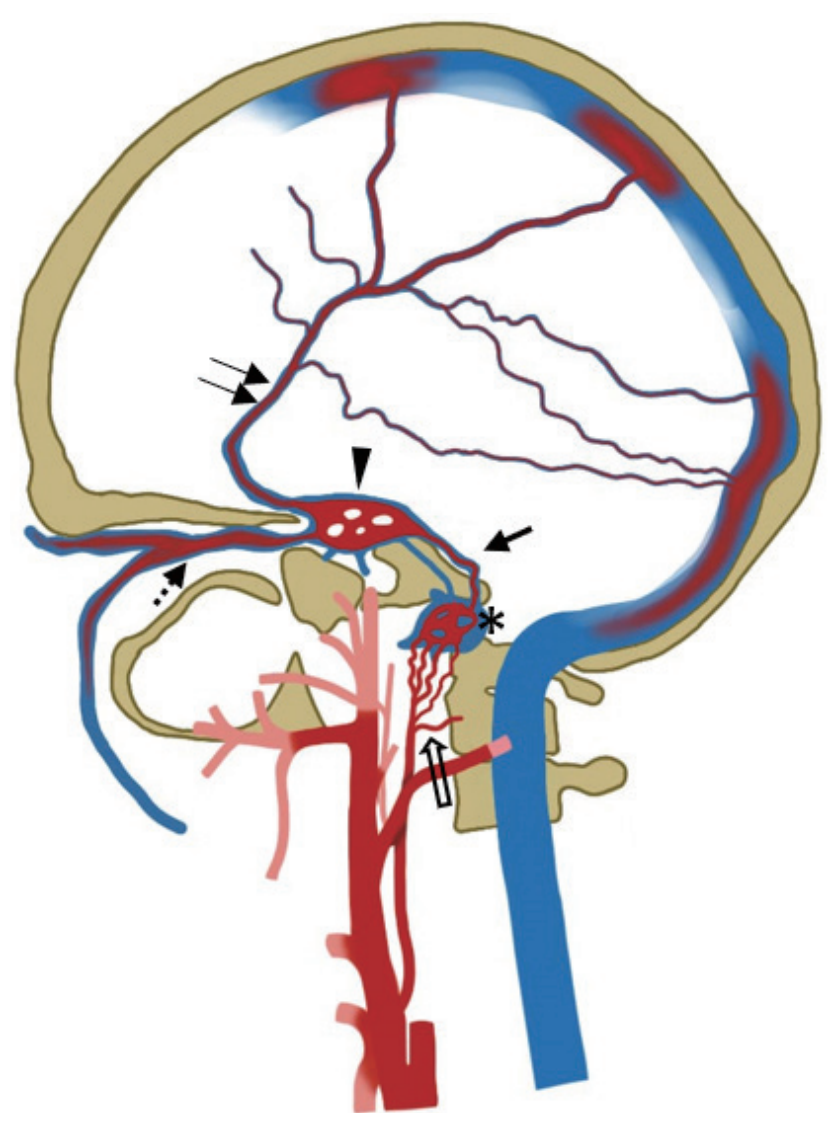

Fig. 2. Diagrammatic illustration of type 2 hypoglossal canal dural arteriovenous fistula with the fistula point in the anterior condylar confluence (asterisk). Dominant retrograde venous drainage to the inferior petrosal sinus (arrow) $\rightarrow$ cavernous sinus (arrowhead) $\rightarrow$ superior ophthalmic vein (dashed arrow), with cortical venous reflux (double arrow). Arterial feeders are depicted from the neuromeningeal division of the ascending pharyngeal artery (open arrow). of the cannula inside the SOV. Then, a Marathon $1.5 \mathrm{~F}$ microcatheter (Medtronic, Irvine, CA, USA) and 0.014-inch Traxcess micro-guidewire (Microvention, Aliso Viejo, CA, USA) combination was navigated via right cavernous sinus, IPS, and advanced into the ACV. Initially, the catheter tip was placed inadvertently into the basilar plexus due to overlapping of the veins in the roadmap, which was identified in Xper CT (Fig. 1E). Subsequently, the catheter was repositioned and a safe position of the catheter tip inside the fistulous pouch was confirmed with Xper CT again (Fig. 1F). Occlusion of the ACV was performed with Barricade platinum coils (Balt, Irvine, CA, USA) under a 2D biplane roadmap. Control angiography revealed complete obliteration of the fistulous shunt (Fig. 1H). Post-embolisation Xper CT demonstrated the coils confined to the right HC (Fig. 1G). The microcatheter was removed and hemostasis of the puncture site was achieved with gentle finger pressure. The clinical course was uneventful without any new neurologic deficit. The eye symptoms and third nerve palsy had completely resolved at the 3-month follow-up visit.

\section{DISCUSSION}

Most DAVFs are considered to be acquired, although the exact etiology is unknown. DAVFs account for $10-15 \%$ of intracranial vascular malformations, and 3-4\% of those occur in the HC, known as ACC DAVF or HCDAVF. The clinical manifestations are variable and depend on the site and venous drainage pathway. Our patient primarily presented with ocular and periorbital symptoms with raised intraocular pressure, features mimicking cavernous sinus DAVFs.

Various therapeutic methods can be used to treat ACC DAVFs. Transarterial embolization is considered to be less effective and is associated with a substantial risk of lower cranial nerve palsies (9th-12th) and embolic stroke, particularly when a liquid embolic agent is used. ${ }^{3}$ Transarterial polyvinyl alcohol particle embolization also carries a higher recurrence rate, although it may help to reduce fistula flow. Surgery may be indicated in patients with Type 3 HCDAVFs with eloquent or poorly accessible feeders. ${ }^{4}$

Transvenous embolization appears to be the most effective treatment for eliminating ACC DAVFs when venous access is available, with a clinical success rate of $91 \%$ and a lower therapeutic risk (permanent morbidity 2.9\%). ${ }^{4}$ In most cases, a microcatheter is navigated to the ACC/ACV through 
ipsilateral trans-jugular access. In some cases, a transvenous approach via the ipsilateral IJV may be impossible because of hypoplasty, tortuosity, or occlusion of IPS. In those situations, a crossover transvenous route through the opposite IJV and IPS may be feasible. ${ }^{5}$ Alternatively direct surgical access to SOV is a possible option for TVE. In the majority of cases, coils are used to occlude the fistulous pouch at the ACC (92.7\%), and other embolic agents were Onyx (2.9\%) or Onyx and coils (2.9\%). ${ }^{4}$

Our management of choice for ACC DAVF is transvenous embolization through the IJV and IPS. This case poses a therapeutic challenge as it belongs to type 2 HCDAVF without antegrade drainage via the IJV. The ACC must be accessed via the ipsilateral cavernous sinus, as there is no connection between ACC and IJV. In the current case, the right IPS could not be recanalized and the right cavernous sinus could not be accessed through the left cavernous sinus due to the presence of multiple tiny channels in the intercavernous sinus. SOV could not be accessed through facial veins, as they were draining into small tortuous veins rather than a single major vein. Direct cannulation of the SOV via surgical cutdown is a widely used technique, but it may be complicated. It may injure the superior root of the fifth cranial nerve, causing forehead numbness and it may also cause levator muscle palsy resulting in ptosis. ${ }^{6}$ So a percutaneous approach to the SOV or cavernous sinus is an alternative solution. As direct cavernous sinus puncture has its inherent risks, we opted for direct percutaneous SOV puncture.

White et al. ${ }^{7}$ successfully treated 8 cases of cavernous sinus dural AV fistula by direct cavernous sinus or inferior ophthalmic vein puncture without the need for a surgical cut-down. Percutaneous venous puncture carries some advantages over direct surgical exposure like lower risk of infections, better cosmetics, and the need for an ophthalmic surgeon in the latter. The direct cavernous sinus puncture also carries some potential risks, including ocular perforation, damage to the II, III, IV, V, and VI cranial nerves, intraorbital hemorrhage, and injury to the cavernous ICA. As an alternative, the direct-puncture approach of the extraconal portion of SOV provides direct access without the requirement for surgical exposure, eliminating the risks in direct cavernous sinus puncture. $^{8}$ The extraconal component of the SOV is thicker and safer than the intraconal segment. Also, when SOV is the predominant draining vein, it will get more dilated and thicker over time, making puncture easier and safer. Since the extra-conal portion of the SOV is superficial, hemostasis can also be achieved easily by applying direct gentle pressure. ${ }^{9}$

The ideal site for SOV puncture is the supero-medial aspect of the orbit, where superior and inferior roots converge to form SOV near the trochlea. However, in our case, peripheral roots and the anterior portion of the first segment of SOV were tortuous and small; hence, we chose the hypertrophied anterior apsidal vein tribute of the SOV for vascular access. The anterior apsidal vein drains into the posterior aspect of the first segment, which marks the junction of extra and intraconal portions of the SOV. ${ }^{9}$ Direct SOV puncture may precipitate venous thrombosis and re-routing of the drainage routes, leading to worsening of orbital symptoms or cerebral venous congestion. However, these complications can be minimized by safe puncture methods and adequate heparinization. $^{10}$

The 3D digital subtraction angiography method helps us for the exact identification of the fistulous point and venous drainage pathway, including draining vein stenosis in relation to bony structures of the skull base. Awareness of the complex venous drainage at the skull base is vital for treatment planning and for understanding the symptoms of ACC DAVFs. In this case, the microcatheter tip was inadvertently placed in the basilar plexus initially, which was repositioned into the fistulous pouch after confirming with the Xper CT. Precise localization of the shunt point by 3D RA and performing Xper CT during the procedure may enable exact placement of the coils within the fistulous pouch and prevent coil overpacking.

\section{CONCLUSION}

HCDAVFs are rare and may exhibit complex venous drainage patterns. Knowledge of the complex venous anatomy is essential for planning an alternative transvenous route if the standard approach is not feasible. After failed attempts through jugular access, our patient was treated by a unique percutaneous direct puncture approach of the SOV, achieving complete obliteration of the fistula. Xper CT is an excellent tool for identifying the exact site of the fistula as well as for confirming the safe position of the catheter tip for successful occlusion of this complex dural AV fistula.

\section{Fund}

None. 


\section{Ethics Statement}

This study was approved by the ethics committee of GKNM hospital. Informed consent for publication was obtained from the patient.

\section{Conflicts of Interest}

The authors have no conflicts to disclose.

\section{Author Contributions}

Concept and design: RP. Analysis and interpretation: HK. Data collection: SK. Writing the article: SK. Critical revision of the article: BK and NK. Final approval of the article: RP. Overall responsibility: SK.

\section{ORCID}

Rasmiranjan Padhi: https://orcid.org/0000-0001-7419-6112

Sathish Kandasamy: https://orcid.org/0000-0002-8870-666X

Balasenthil Kumaran: https://orcid.org/0000-0002-5956-0542

Naci Kocer: https://orcid.org/0000-0003-0884-6492

Harshith Karmadhari: https://orcid.org/0000-0002-8017-4240

\section{REFERENCES}

1. Choi JW, Kim BM, Kim DJ, Kim DI, Suh SH, Shin NY, et al. Hypoglossal canal dural arteriovenous fistula: incidence and the relationship between symptoms and drainage pattern. J Neurosurg 2013;119:955-960

2. Ernst $R$, Bulas R, Tomsick T, van Loveren $H$, Aziz KA. Three cases of dural arteriovenous fistula of the anterior condylar vein within the hypoglossal canal. AJNR Am J Neuroradiol 1999;20:20162020

3. Lv X, Jiang C, Zhang J, Li Y, Wu Z. Complications related to percutaneous transarterial embolization of intracranial dural arteriovenous fistulas in 40 patients. AJNR Am J Neuroradiol 2009;30:462-468

4. Spittau B, Millán DS, El-Sherifi S, Hader C, Singh TP, Motschall E, et al. Dural arteriovenous fistulas of the hypoglossal canal: systematic review on imaging anatomy, clinical findings, and endovascular management. J Neurosurg 2015;122:883-903

5. Manabe H, Hasegawa S, Takemura A, Shafiqul IM, Ito C, Nagahata M. Contralateral inferior petrosal sinus approach for transvenous embolization of a dural arteriovenous fistula at isolated jugular bulb. Technical case report. Minim Invasive Neurosurg 2003;46:366-368

6. Dye J, Duckwiler G, Gonzalez N, Kaneko N, Goldberg R, Rootman D, et al. Endovascular approaches to the cavernous sinus in the setting of dural arteriovenous fistula. Brain Sci 2020;10:554

7. White JB, Layton KF, Evans AJ, Tong FC, Jensen ME, Kallmes $D F$, et al. Transorbital puncture for the treatment of cavernous sinus dural arteriovenous fistulas. AJNR Am J Neuroradiol 2007;28:1415-1417

8. Trivelato FP, Manzato LB, Filho PMM, Ulhôa AC, Vanzin JR, Abud DG, et al. Transorbital cavernous sinus direct puncture: alternative to treat dural arteriovenous fistula. Clin Neuroradiol 2018;28:55-61

9. Kurata A, Suzuki S, Iwamoto K, Miyazaki T, Inukai M, Abe K, et al. Direct-puncture approach to the extraconal portion of the superior ophthalmic vein for carotid cavernous fistulae. Neuroradiology 2009;51:755-759

10. Shinoda N, Mori M, Tamura S, Korosue K, Kose S, Imai H, et al. A patient with a cavernous sinus dural arteriovenous fistula in whom direct puncture of the superior ophthalmic vein led to rapidly progressing thrombosis and postoperative non-arteritic ischemic optic neuropathy: pathogenesis with respect to a drainage route. JNET 2019;13:221-227 